\title{
The reliability of multistory buildings with the effect of non-uniform settlements of foundation
}

\author{
Rafik Al' Malul' 1,*, and Michail Gadzhuntsev ${ }^{1}$ \\ ${ }^{1}$ Moscow State University of Civil Engineering, Yaroslavskoe shosse, 26, Moscow, 129337, Russia
}

\begin{abstract}
The issue is the evaluation of reliability of construction considering the influence of the variation of the support settlement, which is changing during the lifetime of constructions due to the consolidation process of the ground. Recently, the specialists give special emphasis to the necessity to develop the methods for the estimation of reliability and durability of structures. The problem, the article considers, is the determination of the reliability of multistory buildings with non-uniform changing-in-time sediments caused by the consolidation process in soils. Failure of structures may occur before the draft reaches it's stabilizing value, because of the violations of the conditions of normal use.
\end{abstract}

\section{Introduction}

The support settlement has a great effect on the distribution of stresses and deformations of the structure. The most dangerous effects on the behavior of the structure is produced by the difference of the settlement of separate parts of the structure's foundations, that difference causes some extra stresses and deformations of the structure's elements. It is known that the settlement (sagging) depends on the kind of the basement soil and the state of stress in the grounds the foundation consists of.

Let us consider the example: statically indeterminate truss which is situated in the layer of the soil.

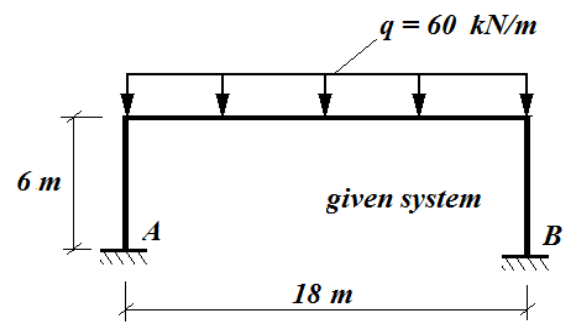

Fig.1 The truss loaded uniformly

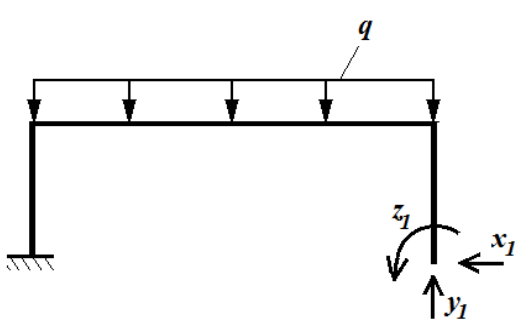

Fig.2 The main system

By the removal of one support, (B), the system is made statically determinate. Take the force of the interaction between the beam of the truss and the support (B) as the main unknown variable. The displacement of the point $(B)$ in the main system in the

\footnotetext{
*Corresponding author: almalyul@yandex.ru
} 
direction of the unknown force $\mathrm{x}_{1}(\mathrm{t})$, the force was caused only by the imposed load, is denoted with $\Delta_{1 p}$, and the displacement of the same point (B) in the direction of the unit force $\mathrm{x}_{1}(\mathrm{t})=1$, denote with $\delta_{11}$.

The values $\Delta_{1 p}$ and $\delta_{11}$ do not depend on time but on the elasticity of the system. They can be determined by the well-known methods of frame-analysis.

The strain compatibility condition for the given statically indeterminate system can be written that way:

$$
\Delta_{1 p}+\delta_{11} x_{1}(t)+s_{1}\left[x_{1}(t), t\right]=0
$$

$s_{1}\left[x_{1}(t), t\right]$ - the function (yet unknown), which characterize the law of the variation of the supports` $(B)$ settlement due to the unknown force $x_{1}(t)$.

The hydrodynamic stress in the soil skeleton, as we know, is determined by the integration of the equation:

$$
\frac{\partial P_{z}}{\partial t}=c^{2} \frac{\partial^{2} P_{z}}{\partial z^{2}}
$$

with the following boundary limits:

$$
\begin{aligned}
& t=0 \text { и } 0 \leq z \leq h_{0}, P_{z}=0: z=0 \\
& t=0 \text { и } z=h_{0}, P_{z}=P(t)=\frac{x_{1}(t)}{F}
\end{aligned}
$$

Solving the equation (2) with the Fourier's method, we have:

$$
P_{z}(t)=p(t)-\frac{4}{\pi} \sum_{k=1,3,5}^{\infty} \frac{1}{k} e^{\lambda_{k}^{2} t} \sin \frac{k \pi z}{h_{0}}\left[p(0)+\int_{0}^{t} p^{\prime}(\xi) e^{\lambda_{k}^{2} \xi} d \xi\right]
$$

here:

$\lambda_{k}^{2}=\frac{k^{2} \pi^{2}}{h^{2}} \mathrm{c}^{2}$

$c_{2}$ - the soil consolidation coefficient, the expression for it: $\mathrm{c}^{2}=k_{0}^{2} \frac{1+\xi_{1}}{\Delta \alpha}$

here:

$k_{0}$ - the coefficient of filtration, and $k_{0}=$ const

$\xi_{1}$ - the porosity mid-index of the soil;

$\alpha$ - the compacting factor under the compression of the soil;

$\Delta$ - the volume weight of water

$$
H(t)=\frac{1}{\Delta}\left[P(t)-P_{z}(t)\right]
$$

The expression for the settlement (sagging) of the soil layer is

$$
s_{1}(t)=\frac{8 k_{0}}{\Delta h_{0}}\left[p(0) \sum_{k=1,3,5}^{\infty} \frac{1-e^{-\lambda_{k}^{2} t}}{\lambda_{k}^{2}}+\sum_{k=1,3,5}^{\infty} \int_{0}^{t} d \tau \int_{0}^{\tau} p^{\prime}(\xi) e^{\lambda_{k}^{2}(\tau-\xi)} d \xi\right]
$$

After changing the order of integration in the last summand, we have:

$$
\sum_{k=1,3,5}^{\infty} \int_{0}^{t} d t \int_{0}^{\tau} p^{\prime}(\xi) e^{-\lambda_{k}^{2}(\tau-\xi)} d \xi=\sum_{k=1,3,5}^{\infty} \frac{1}{\lambda_{k}^{2}} \int_{0}^{t} p^{\prime}(\xi)\left[1-e^{-\lambda_{k}^{2}(t-\xi)}\right] d \xi
$$

After partial integration of the right part: 


$$
\begin{aligned}
\sum_{k=1,3,5}^{\infty} \int_{0}^{t} d \tau \int_{0}^{\tau} p^{\prime}(\xi) e^{-\lambda_{k}^{2}(\tau-\xi)} d \xi & \\
= & -p(0) \sum_{k=1,3,5}^{\infty} \frac{1-e^{-\lambda_{k}^{2} t}}{\lambda_{k}^{2}}+\sum_{k=1,3,5}^{\infty} e^{-\lambda_{k}^{2}(t-\xi)} p^{\prime}(\xi) d \xi
\end{aligned}
$$

Substitute (5) in (7), so we have:

$$
s_{1}(t)=\frac{8 k_{0}}{\Delta h_{0}} \int_{0}^{t} p^{\prime}(\xi) \sum_{k=1,3,5}^{\infty} e^{-\lambda_{k}^{2}(t-\xi)} d \xi
$$

Make a note that

$F$ - the square of the foot of the support $(B)$.

$$
P(t)=\frac{x_{1}(t)}{F}
$$

Finally, we get the following expression for $s_{1}(t)$ :

$$
s_{1}(t)=\frac{8 k_{0}}{\Delta h_{0} F} \int_{0}^{t} x_{1}(\xi) \sum_{k=1,3,5}^{\infty} e^{-\lambda_{k}^{2}(t-\xi)} d \xi
$$

The expression (9) gives us the expression for the variation of the support (B) settlement for our statically indeterminate system as a function of the time $t$ and the value of reaction $x_{1}(t)$.

Substitute $s_{l}(t)$ from (9) in the strain continuity equation (1)

$$
\Delta_{1 p}+\delta_{11} x_{1}(t)+\frac{8 k_{0}}{\Delta h_{0} F} \int_{0}^{t} x_{1}(\xi) \sum_{k=1,3,5}^{\infty} e^{-\lambda_{k}^{2}(t-\xi)} d \xi=0
$$

divide the equation (10) into $\delta_{11}$, with $t=0$, so we have:

$$
\begin{gathered}
x_{1}(0)=-\frac{\Delta_{1 p}}{\delta_{11}}=x_{1}^{0} \\
x_{1}(t)=x_{1}^{0}-\frac{8 k_{0}}{\Delta h_{0} F \delta_{11}} \int_{0}^{t} x_{1}(\xi) e^{-\lambda_{k}^{2}(t-\xi)} d \xi
\end{gathered}
$$

Let us introduce some notations:

$$
\frac{8 k_{0}}{\Delta h_{0} F \delta_{11}}=\beta, \quad \sum_{k=1,3,5}^{\infty} e^{-\lambda_{k}^{2}(t-\xi)}=k(t-\xi)
$$

then the expression (11) turns into:

$$
x_{1}(t)=x_{1}^{0}-\beta \int_{0}^{t} x_{1}(\xi) k(t-\xi) d \xi
$$


So, the analysis of statically indeterminate systems in case of combined action: the load (q) and the settlement of one of it's supports, variative in time, is reduced to the solution of the integral Volterra equation, that is (13). If

$$
\begin{gathered}
k(t-\xi)=\sum_{k=1,3,5}^{\infty} e^{-\lambda^{2} \pi^{2}\left(\frac{c}{n_{0}}\right)^{2}(t-\xi)} \\
k(z)=\sum_{k=1,3,5}^{\infty} e^{-\lambda^{2} \pi^{2} z} \\
z=\left(\frac{c}{n_{0}}\right)^{2}(t-\xi)
\end{gathered}
$$

Use the integral equation (13), so

$$
x_{1}(t)=x_{1}^{0}\left[\frac{1}{1+b}-b \sum_{k=1}^{\infty} \frac{e^{\lambda_{k} t} \sin 2 \lambda_{k}}{\lambda_{k}\left(\cos ^{2} \lambda_{k}+b\right)}\right]
$$

here:

$$
\begin{aligned}
& x_{1}(0)=x_{1}^{0}, \quad b=\frac{\beta}{2}\left(\frac{n_{0}}{2}\right)^{2}, \quad x_{1}(\infty)=\frac{x_{1}^{0}}{1+b}, \\
& \sum_{k=1}^{\infty} \frac{\sin 2 \lambda_{k}}{\lambda_{k}\left(\cos ^{2} \lambda_{k}+b\right)}=-\frac{1}{1+b}
\end{aligned}
$$

Come back to our example - statically undetermined truss, shown in fig. 1 (the main system is shown in fig.2). Set the physical and mechanical properties for the foundation and the construction

$$
\begin{aligned}
& F=49 \mathrm{~m}^{2}-\text { base area; } \\
& E J=9 \cdot 10^{3} \mathrm{~kg} \cdot \mathrm{cm}^{2}-\text { the stiffness of the section; } \\
& q=60 \mathrm{~kg} / \mathrm{m} \text { - the load; } \\
& k_{0}=2,2 \cdot 10^{7} \mathrm{~cm} \text { per second - the coefficient of filtration; } \\
& \xi_{1}=1,368-\text { the porosity mid-index of the soil; } \\
& \mathrm{c}^{2}=k_{0} \frac{1+\xi_{1}}{\Delta \alpha}=0,253 \cdot 10^{-2} \mathrm{~cm}^{2} / \text { second - the compacting factor of the soil; } \\
& h_{0}=4 \mathrm{~m}-\text { the height of the soil layer; } \\
& \beta=6,24 \cdot 10-7 \text { gramm per second; } \\
& \Delta=5,17 .
\end{aligned}
$$

Table 1 contains the values of bending moments in the truss.

Table 1. values of bending moments in the truss

\begin{tabular}{|l|l|l|l|l|l|l|l|}
\hline $\mathrm{t}$ & & & & & & & \\
\hline $\mathrm{t}=1$ day & 61,02 & 96,36 & 96,36 & 40,5 & $-49,77$ & $-49,77$ & $-85,11$ \\
\hline $\mathrm{t}=7$ days & 86,88 & 89,28 & 89,28 & $-40,5$ & $-47,52$ & $-47,52$ & $-49,92$ \\
\hline $\mathrm{t}=3$ months & 55,51 & 90,85 & 90,85 & $-40,5$ & $-44,51$ & $-44,51$ & $-79,85$ \\
\hline $\mathrm{t}=1$ year & 54,78 & 90,54 & 90,54 & $-40,5$ & $-44,1$ & $-44,1$ & $-79,86$ \\
\hline $\mathrm{t}=\infty$ & 54,78 & 90,59 & 90,57 & 40,6 & $-44,3$ & $-44,35$ & $-79,88$ \\
\hline
\end{tabular}


The most bending moment in the framework is

$$
M_{\max }=162 q\left[\frac{1}{1+b}-b e^{-s t} \frac{\sin 2 \lambda_{1}}{\lambda_{1}\left(\cos ^{2} \lambda_{1}+b\right)}\right]+18,33 q-6,11 q-162 q
$$

Let us introduce some notations:

$$
\begin{aligned}
& M_{\text {max }}=\left(\frac{162}{1+\beta}+18,33-6,11-162\right) q \\
& \mathrm{D}=\frac{162 \mathrm{qb} \cdot \sin 2 \lambda_{1}}{\lambda_{1}\left(\cos ^{2} \lambda_{1}+\mathrm{b}\right)}
\end{aligned}
$$

The situation is that the limits of the fail-safe behavior area is determined by the condition:

$$
M_{\text {max }}=M_{0}-D e^{s, t} \leq M_{a c c}
$$

( $\mathrm{M}_{\mathrm{acc}}$ - the acceptable value of the moment)

The condition could be transformed to

$$
z=\frac{M_{0}-M_{\text {доп }}}{D}=e^{s, t}
$$

Take only the first member of the raw in (17) and prelogarithmic both parts of (17), so we get:

$$
\ln z=\omega=s, t
$$

here

$s_{1}=-\frac{\lambda_{1}^{2}}{\alpha_{0}^{2}}$

The probability features of $\omega$ depends on the random properties of all the variables the function $z$ depends on. The limits of the fail-safe behavior area depends on the random value $z=z_{0}\left(\omega=\omega_{0}\right)$, which has mathematical expectation $\overline{\omega_{0}}$ and the dispersion $\sigma^{2}\left(\omega_{0}\right)$. Suppose that the failure happens then $M_{\max }=M_{a c c}$, the probabilistic properties of $z_{0}\left(\omega_{0}\right)$ are defined only by the probabilistic properties of $M_{\max }^{0}$, and the probabilistic properties of $\mathrm{s}$ are defined by the variation of the consolidation coefficient.

The exact time of failure corresponds to the point where the stochastic process (21) and the probable limits of the no- failure behavior area cross. Suppose that then the failure happens $s=\omega_{0} / T$, the characters $s$ and $\omega_{0}$ are independent and random and that they have a truncated Gaussian distribution in the intervale $(0, \infty)$. This is so, for example, then the random value of $\mathrm{z}$ has a logarithmically normal distribution of the random value $\mathrm{T}$, such as

$$
F(t)=\alpha_{\omega_{0}} \alpha_{s} \int_{0}^{\infty}\left[\int_{\omega_{0} / t}^{\infty} f(s) d s\right] f\left(\omega_{0}\right) d \omega_{0}
$$

here $\alpha_{\omega 0}, \alpha_{s}$ - normalizing multiplier factors of that distribution. 


$$
\begin{gathered}
\alpha_{\mathrm{n}}=\left[0,5+\Phi\left(\frac{\mathrm{m}_{\mathrm{n}}}{\sigma_{\mathrm{n}}}\right)\right]^{-1} \\
\mathrm{n}=\omega_{0}, \mathrm{~s}
\end{gathered}
$$

The expression for the density of distribution for no-failure behavior is that:

$$
f(t)=\frac{d F(t)}{d t}
$$

Then, in accordance with (4), it can be written down:

$$
\frac{d}{d t} \int_{\omega_{0} / t}^{\infty} f(s) d s=\frac{\omega_{0}}{\sigma_{(s)} t^{2} \sqrt{2 \pi}} \exp \left[\frac{1}{2}\left(\frac{\omega_{0}}{\sigma_{(s)} t}-\frac{m s}{\sigma_{(s)}}\right)^{2}\right]
$$

Substitute (22) into (24) and take into account (25), then we get the expression for the density distribution for the time of no-failure behavior of the construction:

$$
\begin{gathered}
f(t)=\frac{\alpha_{\omega 0} \alpha_{s} \exp \left[0,5\left(\beta^{2} / \sigma+\alpha^{2}\right)\right]}{2 \pi\left(t^{2}+\sigma^{2}\right)} \\
+\frac{\alpha_{\omega 0} \alpha_{s}\left(\beta t+\alpha \sigma^{2}\right)}{\sqrt{2 \pi\left(t^{2}+\sigma^{2}\right)}} \exp \left[-\frac{(\beta-\alpha t)^{2}}{2\left(t^{2}+\sigma^{2}\right)}\right][0,5 \\
\left.+\Phi \frac{\beta t+\alpha \sigma^{2}}{\sigma \sqrt{t^{2}+\sigma^{2}}}\right] \\
\sigma=\sigma \frac{\omega_{0}}{\sigma(s)} ; \quad \beta=\frac{\overline{\omega_{0}}}{\sigma(\mathrm{s})} ; \alpha=\frac{\overline{\mathrm{s}}}{\sigma(\mathrm{s})}
\end{gathered}
$$

The reliability function of the system is determined by the following expression:

$$
P(t)=1-\int_{0}^{t} f(t) d t
$$

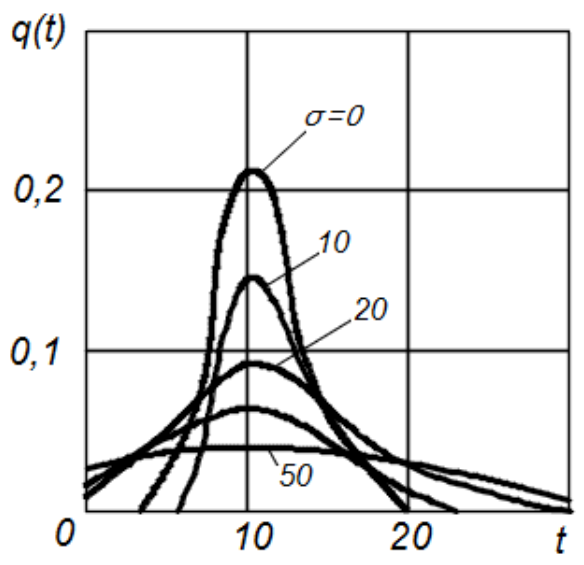

Fig.3 The distribution density of non-failure work 


\section{Conclusions}

The variation of the support settlement has a significant effect on the distribution of moments in the left vertical pier and in the beam of the truss. The resultant expression (26) for the density distribution of the time of no-failure behavior corresponds to the physical meaning of the task. The rate of accumulation of settlement decreases over time. We can find from (27) that the rate of the decrease of the reliability function is the most of all at the start moment and decreases gradually. For example, let the coefficient of variation, the consolidation coefficient be 0,2 and $\beta$ be 50 , then we get, - for various values of $\sigma$ in accordance with (26), - different curves describing the density distribution for the time of no-failure behavior.

We should make a note that $t=0, f(t) \neq 0$.

Fig. 3 shows that the density distribution of the time of no-failure behavior increases from the constant value $t=t_{i}$ and, with increasing value of $t$, the density distribution decreases.

\section{References}

1. Al Malul R.M., PhD Thesis

2. N.H Arutyunyan Some problems of the creep theory (Moscow, 2006)

3. A.R. Rzhanitsyn The theory of analysis for the reliability of engeneering constructions. M.; StroyIzdat, 1978, 240 c.

4. V.D. Raizer, The reliability theory of constructions. Association of the Civil Engineering Universities, Moscow, 2010, 382 p.

5. V.D. Raizer Reliability Optimization for Pipelines to Corrosion Wear, Proc.of ASCE conference Advances in Pipeline Engineering And Construction. Pipelines 2001. San-Diego, CA, US, 2001.

6. Russian State Standard SNIP 2.03.01.84 